\title{
Análise sobre a saúde mental de mulheres profissionais da educação no cenário pandêmico
}

\author{
Analysis of the mental health of women education professionals in the pandemic scenario \\ Análisis de la salud mental de las mujeres profesionales de la educación en el escenario de una
} pandemia

Recebido: 14/07/2021 | Revisado: 22/07/2021 | Aceito: 08/09/2021 | Publicado: 11/09/2021

Bárbara Moraes de Mello

ORCID: https://orcid.org/0000-0002-0421-7827

Universidade Federal de Campina Grande, Brasil

E-mail:barbaramello@hotmail.com

Antônio Levi Sampaio de Araújo

ORCID: https://orcid.org/0000-0002-8880-0736

Faculdade Santa Maria, Brasil

E-mail: lev_sampaioaa@outlook.com

Jefferson Washington de Souza Rodrigues

ORCID: https://orcid.org/0000-0002-3805-0607

Faculdade Santa Maria, Brasil

E-mail: jefferson.4545@hotmail.com

Auzenir de Oliveira Abrantes Monteiro

ORCID: https://orcid.org/0000-0003-0363-620X

Universidade Federal de Campina Grande, Brasil

E-mail: auzenirabrantes@gmail.com

Myria Juscilania Maraco Silva

ORCID: https://orcid.org/0000-0002-0733-5482

Universidade Federal do Rio Grande do Norte, Brasil

E-mail: myriamaraco84@gmail.com

Gleiciane Ingrith Lins de Morais

ORCID: https://orcid.org/0000-0001-5739-4088

Universidade Federal do Rio Grande do Norte, Brasil

E-mail: gleiciane_ingrith@hotmail.com

Maria Emanuele do Rego Santos

ORCID: https://orcid.org/0000-0003-1488-8656

Universidade Federal do Rio Grande do Norte, Brasil E-mail:emanuelersantos@gmail.com

Itamara Lígia Rodrigues Vieira

ORCID: https://orcid.org/0000-0003-1670-7505

Universidade Federal do Rio Grande do Norte, Brasil E-mail-itamarailrvieira@gmail.com

Agne Bárbara da Silva Costa Dantas

ORCID: https://orcid.org/0000-0001-6652-5748

Universidade Federal do Rio Grande do Norte, Brasil E-mail: agne.dantas.012@ufrn.edu.br

José Cândido da Silva Nóbrega

ORCID: https://orcid.org/0000-0002-0976-3763

Universidade Federal de Campina Grande, Brasil E-mail: jcandidosn@uol.com.br

Manoel Marques de Souto Nóbrega Filho ORCID: https://orcid.org/0000-0003-4512-2733

Faculdade de Ciências Médicas da Paraíba, Brasil

E-mail: manoelmarquesnobrega@gmail.com

\section{Resumo}

A pandemia da Covid-19, apresenta-se como um dos maiores desafios sanitários deste século, gerando uma crise sem precedentes na saúde pública do Brasil, e a partir da proliferação desse vírus, se propagou um pânico generalizado onde milhares de pessoas foram acometidos por distúrbios psicológicos, tendo como principal alvo o público feminino. Diante do estado de calamidade pública, o Ministério da Educação decretou novas portarias, e foi necessário que as instituições de ensino e seus educadores criassem estratégias para promoção da educação à distância. Tendo em vista essa problemática, e para atingir o objetivo geral dessa pesquisa, será feito um estudo a partir da estruturação de uma revisão da literatura e análise de dados, sobre os impactos da pandemia na saúde mental, em especial, das mulheres que trabalham na educação. Para a realização desse estudo, será empregado o método e 
abordagem dedutivo, em nível de profundidade essa pesquisa será constituída por meio da pesquisa exploratória através da coleta de dados bibliográficos de natureza qualitativa e descritiva. Os resultados encontrados apontam um impacto psicológico na saúde das mulheres que trabalham na educação, provenientes de problemas emocionais, decorrente da situação provocada pela pandemia da Covid-19, onde muitas vezes precisam lhe dar com a pressão dos sistemas de ensino, aliado a dupla jornada de trabalho, podendo essa situação configurar numa fragilidade psicológica, contribuindo para um agravamento da saúde mental. Nesse sentido, é preciso pensar, planejar e implementar ações de suporte à saúde mental com atendimentos psicossociais mais frequentes para essas profissionais.

Palavras-chave: Pandemia; Saúde mental; Mulheres educadoras.

\begin{abstract}
The Covid-19 pandemic presents itself as one of the greatest health challenges of this century, generating an unprecedented crisis in public health in Brazil, and from the proliferation of this virus, a widespread panic has spread, where thousands of people have been affected by disturbances. psychological, having as main target the female audience. Faced with the state of public calamity, the Ministry of Education decreed new ordinances, and it was necessary for educational institutions and their educators to create strategies to promote distance education. In view of this problem, and to achieve the general objective of this research, a study will be carried out based on the structuring of a literature review and data analysis, on the impacts of the pandemic on mental health, especially on women who work in the education. To carry out this study, the deductive method and approach was used, in depth this research was constituted through exploratory research through the collection of bibliographic data of a qualitative and descriptive nature. The results found point to a psychological impact on the health of women who work in education, arising from emotional problems, resulting from the situation caused by the Covid-19 pandemic, where they often need to deal with the pressure of education systems, combined with the double journey of this situation may lead to psychological fragility, contributing to a worsening of mental health. In this sense, it is necessary to think, plan and implement actions to support mental health with more frequent psychosocial care for these professionals.
\end{abstract}

Keywords: Pandemic; Mental health; Women educators.

\title{
Resumen
}

La pandemia del Covid-19 se presenta como uno de los mayores desafíos de salud de este siglo, generando una crisis de salud pública sin precedentes en Brasil, y a partir de la proliferación de este virus se ha extendido un pánico generalizado, donde miles de personas se han visto afectadas por disturbios. psicológico, teniendo como principal objetivo al público femenino. Ante el estado de calamidad pública, el Ministerio de Educación decretó nuevas ordenanzas, y era necesario que las instituciones educativas y sus educadores crearan estrategias para promover la educación a distancia. Ante esta problemática, y para lograr el objetivo general de esta investigación, se realizará un estudio a partir de la estructuración de una revisión de la literatura y el análisis de datos, sobre los impactos de la pandemia en la salud mental, especialmente en las mujeres que laboran en la educación. Para la realización de este estudio se utilizó el método y enfoque deductivo, en profundidad esta investigación se constituyó a través de una investigación exploratoria a través de la recolección de datos bibliográficos de carácter cualitativo y descriptivo. Los resultados encontrados apuntan a un impacto psicológico en la salud de las mujeres que se desempeñan en la educación, derivado de problemas emocionales, producto de la situación provocada por la pandemia del Covid-19, donde muchas veces necesitan lidiar con la presión de los sistemas educativos, combinada con la El doble recorrido de esta situación puede conducir a la fragilidad psicológica, contribuyendo a un empeoramiento de la salud mental. En este sentido, es necesario pensar, planificar e implementar acciones de apoyo a la salud mental con una atención psicosocial más frecuente para estos profesionales.

Palabras clave: Pandemia; Salud mental; Mujeres educadoras.

\section{Introdução}

A pandemia da Covid-19 causada pelo novo coronavírus, é considerada como um dos maiores desafios sanitários em escala global deste século, só na metade do mês de abril, poucos meses depois do início da epidemia na China em fins de 2019, já haviam ocorrido mais de 2 milhões de casos e 120 mil mortes no mundo. E no Brasil, tinham sido registrados cerca de 21 mil casos confirmados e 1.200 mortes causadas por essa doença (Werneck \& Carvalho, 2020).

Foi a partir da proliferação desse surto, que se propagou um pânico generalizado na população de todo o mundo, onde milhares de pessoas ficaram reféns de distúrbios psicológicos provocados pelo medo das consequências provenientes das infecções e distanciamento social provocado por esse vírus. E consequentemente, houve um aumento significativo de casos de ansiedade, depressão e estresse (Lima et al., 2020). 
Essa nova situação acarretou em uma série de mudanças na vida social, provocadas pela pandemia da Covid-19, e a partir disso, houve uma mudança repentina na vida da população brasileira, como os cuidados permanentes com as medidas de higiene, saúde, isolamento social, fechamento de estabelecimentos comerciais de instituições de ensino entre outras medidas necessárias.

Diante do estado de emergência, e em decorrência do avanço da propagação da pandemia e da alta taxa de transmissibilidade do vírus, o Ministério da Educação decretou novas portarias, e foi necessário que as instituições de ensino e seus educadores criassem estratégias para a promoção da educação à distância, de modo a prover um ensino inovador. (Silva et al., 2020).

Essas mudanças provocaram vários impactos na vida desses profissionais que trabalham na educação que é composto em sua grande maioria por mulheres, as quais tiveram que aprender a utilizar novas tecnologias como ferramenta de trabalho, e tiveram que se adaptar a uma jornada dupla de trabalho em casa, o que causou consequentemente um grande sobrecarregamento de suas atividades.

Dentro dessa problemática, surge a seguinte indagação: como está a saúde mental das mulheres educadoras na pandemia? Pois, nunca um evento trouxe tantos impactos para a rotina de tantas pessoas, com reflexos na relação com os amigos, com a família e, de certo modo, na própria liberdade.

Em frente a tantas incertezas, as emoções afloram, trazendo consigo reações como medo, ansiedade, estresse e outros sentimentos desagradáveis. Pois infelizmente, a fragilidade na saúde mental das educadoras é algo esperado e justificável, em um contexto de crise que afeta a todos.

Para atingir o objetivo geral dessa pesquisa, será realizado um estudo a partir da estruturação de uma revisão narrativa da literatura e análise de dados de pesquisas sobre os impactos da pandemia do novo coronavírus na saúde mental da população, e em especial das mulheres que trabalham na educação. As reflexões aqui problematizadas partirão da leitura e análise dos autores à luz da literatura pertinente à temática da pesquisa, uma vez que esse estudo possa auxiliar no embasamento de debates teórico científicos e colaborativos.

Diante da temática proposta, entende-se que este tema possui grande relevância social, pois em tempos de pandemia a saúde mental da população e, principalmente, da mulher educadora, sofreu alterações decorrente das mudanças proporcionadas pela Covid-19, e apesar dos estudos serem escassos, esse tema merece total atenção dos governos, e das autoridades de saúde, para que se sejam elaboradas medidas psicossociais mais efetivas direcionadas para este público específico.

Tendo em vista a relevância do tema proposto, será realizada uma pesquisa exploratória, que tem por objetivo proporcionar uma maior familiaridade com o problema. Sem a perspectiva de esgotamento do tema, tratar-se-á, sobretudo, acerca dos aspectos mentais em tempo de pandemia. Ademais, se utilizará do método de abordagem dedutivo, uma vez que se buscará os resultados a partir da análise geral até concluir de maneira particular a hipótese. Quanto à natureza da pesquisa, esta será qualitativa, buscar-se-á analisar e interpretar como a pandemia atinge diretamente a saúde mental das pessoas, como forma de chegar à conclusão de que ocorreu não impactos da pandemia na saúde mental da população brasileira.

Quanto ao método de procedimento, atribuir-se-á o método monográfico, partindo de um estudo profundo sobre as $a$ saúde mental no cenário pandêmico, que podendo esta pesquisa ser considerada representativa de muitos outros ou mesmo de todos os casos semelhantes. Além do mais, quanto aos procedimentos técnicos, se delineará o presente estudo por meio de pesquisa bibliográfica, tendo em vista que se buscará, através da renomada e periódicos especializados, a solução para discorrer acerca das mudanças provenientes da Covid-19 afetam a saúde mental das mulheres que trabalham na educação. 


\section{A Pandemia da Covid-19 no Brasil}

A doença respiratória altamente contagiosa da Covid-19 causada pelo novo coronavírus, tem sido um grande desafio para a população brasileira, pois essa doença, foi identificada inicialmente no ano de 2019 na China, e até hoje está disseminada pelo mundo devido a sua alta capacidade de transmissão e mutação e com isso, a doença foi classificada como pandemia pela Organização Mundial da Saúde (OMS).

Para conter essa situação da alta transmissão do vírus, de acordo com especialistas e estudiosos da área da saúde, umas formas mais eficazes de prevenção do contágio, é através da adoção de práticas de higiene e distanciamento social. Ademais, para consubstanciar esse esforço, não deve haver aglomeração de pessoas e, sempre que possível, as pessoas, devem evitar ao máximo sair de casa nesse período (Opas, 2020).

Nesta senda, um dos principais problemas a ser enfrentado é o pouco conhecimento científico que se tem sobre esse vírus, principalmente pela sua alta velocidade de disseminação e capacidade de provocar mortes em populações mais vulneráveis, pois esse é um dos fatores que gera muitas incertezas sobre quais seriam as melhores estratégias a serem utilizadas para o enfrentamento da epidemia em diferentes partes do mundo (Werneck \& Carvalho, 2020).

Outrossim, essa falta de conhecimento sobre o coronavírus torna os desafios ainda maiores no Brasil, justamente por não se saber das características de transmissão num contexto de extrema desigualdade social, onde a maior parte das populações vive em condições precárias de habitação e saneamento, sem acesso sistemático à água e em situação de aglomeração (Werneck \& Carvalho, 2020).

Nesse contexto, devido à insuficiência de conhecimento aliado a acentuada taxa de transmissibilidade e mortalidade do vírus associado a falta de tratamentos mais eficazes, medidas e recomendações preventivas foram instaladas para conter a disseminação da doença para tentar conter o avanço desenfreado do vírus para evitar o amento de casos na população (Peca et, al., 2020).

Para evitar a disseminação desenfreada desse virus, foram aplicadas diversas medidas como: a restrição do convívio representada pelo distanciamento social, o uso obrigatório de máscaras e a higienização frequente das mãos associado a utilização de álcool em gel, essas foram algumas das ferramentas utilizadas, variando de intensidade de acordo com as políticas de saúde de cada governo (Senicato, Azevedo, \& Barros, 2018).

E como consequência, da adoção dessas medidas preventivas que passaram a ser aplicadas para evitar a proliferação do vírus, sentimentos de medo e angústia começaram a aparecer mais acentuados nos indivíduos, os quais, são associados ao anseio de uma possível infecção, tornaram-se gatilhos para manifestações clínicas de psicopatologias (Thampa et al., 2020).

Em suma, a partir desse contexto, verifica-se que, depois da chegada do vírus da Covid-19 no Brasil, houve uma série de mudanças relacionadas principalmente ao convívio, que foram impostas através do isolamento social para conter a disseminação mais rápida do vírus, o que acarretou medo e pânico na maioria das pessoas e consequentemente, houve um grande aumento em relação aos casos de doenças psicológicas.

\section{Saúde Mental na Pandemia}

A carga psicossocial está se tornando cada vez mais exorbitante devido ao agravo da pandemia, à medida que se torna mais necessário o distanciamento social, que é uma das principais estratégias mais eficazes para diminuir o contagio do vírus, por outro lado, essa medida produz efeitos deletérios como sentimento de solidão e desamparo, perda de emprego, prejuízos à saúde física e psíquica, exposição ao adoecimento e morte de amigos e familiares (Kola, 2020).

Esses problemas psicossociais gerados pela disseminação do vírus, tem causado uma série de medo e aversão em fronte a ameaça da doença na população, e em consequência disso, gerou diversos transtornos à saúde e à vida das pessoas, 
além de desencadear uma extensa variedade de problemas psicológicos e transtornos mentais nas pessoas acometidas e não acometidas por esse vírus (Qiu et al., 2020).

Em consequência, o aumento desse surto provocou uma crise de preocupação exagerada, e os sujeitos que são acometidos entram em pânico e sintomas costumam aparecer principalmente por meio de respostas corporais, através da alteração da frequência respiratória, palpitações, e assim por diante, e podem ocasionar um intenso sofrimento psíquico (Machado et, al., 2020).

E foi a partir da proliferação desse surto da Covid-19, que se propagou um pânico generalizado na população de todo o mundo, onde milhares de pessoas ficaram reféns de distúrbios psicológicos provocados pelo medo das consequências provenientes das infecções e distanciamento social provocado por esse vírus. E de forma análoga, houve um aumento significativo de casos de ansiedade, depressão e estresse (Lima et al., 2020).

Dentro desse contexto, um dos principais objetivos do distanciamento social é reduzir as interações a aproximação dos indivíduos em uma mesma comunidade, e para atingir esses objetivos é preciso realizar atos mais drásticos como o fechamento de escolas e locais de trabalho, suspensão de alguns tipos de comércio e o cancelamento de eventos (Aquino et al., 2020).

Entretanto, essas medidas causam bastantes problemas referentes a saúde mental dos indivíduos, e dentro dessa perspectiva, foi feita uma revisão de literatura sobre saúde mental e Covid-19, onde indicou que, do total de 43 artigos selecionados, 33 artigos, ou seja, 77\% sugeriam uma relação entre sintomas de ansiedade e pandemia da Covid-19 (Machado et al., 2020).

É certo uma que uma grande parte desses sintomas de saúde mental, são provenientes da pandemia, e devem ser desenvolvidas estratégias, como prestação de serviços mais eficazes direcionadas para esses indivíduos, com ênfase nos atendimentos psiquiátricos e psicológicos, além da viabilização de incentivo à prática de atividades físicas e meditação de forma regular (Miranda et, al., 2020 p.6).

No entanto, ainda são limitados os estudos que apontem a influência do surto de Covid-19 na saúde mental auxiliados a intervenções vitais para atenuar o crescimento de tal incidência. Pois a saúde mental é tratada como um acontecimento secundário no tocante ao aparecimento do vírus e merece que tenha uma atenção mais especial, principalmente para o público mais afetado psicologicamente devido a sua maior vulnerabilidade.

\section{Análise da Saúde Mental das Mulheres Profissionais da Educação}

De acordo com a divulgação da pesquisa realizada pela equipe do neuropsicólogo Antônio de Pádua Serafim, em matéria veiculada no site da UOL, a pandemia impactou a saúde mental e aspectos comportamentais dos brasileiros, em um estudo realizado entre maio e junho de 2020 com homens e mulheres de várias regiões do País dos 26 Estados brasileiros e do Distrito Federal, mostrou que um número grande de pessoas apresentou, durante a pandemia, sintomas de depressão, ansiedade e estresse.

. Através desse estudo, também foi possível constatar que, as mais afetadas emocionalmente foram as mulheres, respondendo por 40,5\% de sintomas de depressão, 34,9\% de ansiedade e 37,3\% de estresse, essa pesquisa ouviu três mil voluntários. Esses sintomas emocionais estão vinculados principalmente, a vulnerabilidade emocional feminina, que está interligada com as alterações hormonais durante o período menstrual, pré e pós parto e as alterações da menopausa, outro fator que contribui é a desigualdade de gênero, em adição com as sobrecargas trabalhistas e a violência contra a mulher (Souza et al., 2020).

Além disso, alguns dessas mulheres cumprem uma jornada dupla de trabalho, acompanham o desenvolvimento escolar dos filhos e, em decorrência das restrições impostas pela pandemia, mais pessoas permaneceram dentro de casa, além 
das preocupações relacionadas ao próprio vírus iminência de contaminação, necessidade de mudanças de hábitos de higiene, redução de convívio social, familiares adoecidos, entre outros.

Essas mudanças nas relações socias, acarretaram uma série de transformações na vida da população, como os cuidados permanentes com as medidas de higiene e saúde, isolamento social, fechamento de estabelecimentos comerciais de instituições de ensino entre outras medidas necessárias.

Diante do estado de emergência, e em decorrência do avanço da propagação da pandemia e da alta taxa de transmissibilidade do vírus, o Ministério da Educação decretou novas portarias, e foi necessário que as instituições de ensino e seus educadores criassem estratégias para a promoção da educação à distância, de modo a prover um ensino inovador (Silva et al., 2020).

Em face desse novo contexto imposto pelo distanciamento social, e com todas essas alterações na metodologia de trabalho, as mulheres da educação, precisaram modificar sua rotina e trazer a sala de aula para sua casa para realizar seu trabalho em home office, ademais precisaram aprender a lidar com novas ferramentas de ensino para se adequar a essa situação atípica, e da mesma forma foram com os estudantes, tudo isso na intenção de zelar sua vida e de seu próximo (Costa et, al., 2020).

Diante dessa preocupação pesquisadores da Fundação Fiocruz realizaram uma pesquisa que foi publicada no livro: Os impactos sociais da Covid-19 no Brasil: populações vulnerabilizadas e respostas à pandemia. E através desse estudo, foi apontado que tanto as dificuldades como a sobrecarga de trabalho e a exposição ao risco de infecção também são mais marcantes entre as mulheres. E no tocante as mulheres que precisaram executar seu trabalho em casa, essas tiveram que realiza-lo na modalidade conhecida popularmente como home office, e nessa perspectiva a pressão e o acúmulo de responsabilidades colocam em risco a saúde física e mental.

Percebe-se que diante desse cenário pandêmico, os educadores se expõem a diversas situações e pressões das instituições, o que reverbera no adoecimento, principalmente da saúde mental, onde dados alarmantes podem ser observados através da pesquisa realizada pela revista Nova Escola. A pesquisa foi realizada com 9.557 educadores de todo Brasil, e destes $85 \%$ eram mulheres, essa pesquisa foi realizada no primeiro trimestre da pandemia, e quando os profissionais foram questionados sobre a sua relação com saúde mental, 28\% classificaram-na como ruim ou péssima e 30\% como razoável.

Outros fatores que foram apontados pela pesquisa estão relacionados à dupla jornada das mulheres, dividindo-se entre as tarefas domésticas e profissionais, além de, muitas vezes, serem as responsáveis em cuidar das atividades escolares e acompanhar as aulas virtuais dos próprios filhos, faltando tempo para a pratica de atividades de lazer.

Portanto, percebe-se que, além da sobrecarga da dupla jornada de trabalho em sua grande maioria, essas educadoras em muitos casos não têm tempo para praticar atividades que contribuem com a melhora da qualidade de vida, e ajudam a prevenir transtornos mentais, como exercícios físicos e atividades de lazer, os quais são menos prevalentes entre essas mulheres, em decorrência, principalmente, da sobrecarga de funções associadas as sobrecargas de trabalho (Pinho Ps, 2012).

Dessa forma, diante do cenário de mudanças impostas para essas mulheres educadoras através da situação de emergência no Brasil por causa da pandemia da Covid-19, e em função da dupla jornada de trabalho, muitas foram acometidas por doenças psicológicas, e por conseguinte, essas profissionais merecem uma atenção psicossocial mais eficaz de forma continuada por parte dos governantes.

\section{Resultados e Discussão}

Um ponto importante nessa discussão é a vulnerabilidade psicossocial a qual a população em geral, e especialmente, as mulheres que trabalham na educação, estão sujeitas aos impactos fomentados pela crise de uma pandemia, logo, é necessário 
que essas problemáticas sejam destacadas nesse cenário, a fim de buscar estratégias e redes de apoio de psicossociais voltadas para essas profissionais.

Essas estratégias devem ser ancoradas em uma expectativa renovada das práticas preventivas e de promoção da saúde mental, abrindo importantes possibilidades para a discussão epistemológica e ético jurídica em relação ao cuidado da saúde mental, porque a vulnerabilidade se apresenta como uma condição intrínseca de um indivíduo mais propenso, que diante de um evento traumático, gera um transtorno psicológico.

Dentro desse grupo mais propenso, encontram-se as mulheres que trabalham na educação, devido a sua grande porcentagem de prospecção de acometimento de doenças psicológicas que são provenientes de suas atividades, exercidas nesse momento de pandemia, portanto, esse grupo de mulheres merece ser assistida, por uma rede de profissionais habilitados de forma continuada e permanente, que deve ser entendida como um dos pilares prioritários, uma vez que pode fortalecer a rede de resiliência no enfretamento dessa pandemia (Silva et, al,. 2020).

Daí, a importância das redes de atendimentos psicossociais, para prestar ajuda a essas profissionais que precisam de suporte para aguentar essa carga muito elevada de estresse, proveniente de doenças mentais desenvolvidas nesse processo de mudança repentina, aliada a sobrecarga e responsabilidade da readequação, para priorizar a qualidade do ensino imposta pelo Ministério da Educação.

Contudo, mesmo que muitos dos protocolos assistenciais e de intervenção tenham sido desenvolvidos, grande parte dessas profissionais que trabalham diretamente na educação nesse período de Covid-19, não estavam preparadas para lhe dar com as mudanças do ensino, principalmente, durante o início da pandemia, os quais devem receber um atendimento especializado (Xiang Yt, 2020).

Diante desse contexto de mudança, ocasionada pela pandemia na vida dessas mulheres que trabalham na educação, e por não se sentirem preparadas, e ao mesmo tempo tendo que lhe dar com a pressão dos sistemas de ensino, essa situação pode configurar numa fragilidade assistencial, podendo contribuir para um agravamento da saúde mental ainda maior.

Em consequência do avanço da Covid-19, autoridades mundiais, cientistas e profissionais da saúde trabalham incansavelmente em busca métodos de controle e combate dessa doença e, apesar da efetividade dessas ações, os efeitos secundários advindos indiretamente da pandemia estão sendo ofuscados, como o comprometimento da saúde mental do público em geral, em especial do público feminino (Souza et al., 2020).

Diante dessa falta de comprometimento por parte dos governos, é importante salientar sobre a necessidade de se criar estratégias de caráter multiprofissional, que possa abranger e individualizar os fatores secundários que exercem influência diretamente na saúde mental das mulheres que trabalham na educação, que já era afetada por outros fatores antes da pandemia e foi agravado na pandemia por esse conjunto de modificações na convivência social e atividades relacionadas ao trabalho educacional.

\section{Considerações Finais}

Do ponto de vista da saúde mental, e, através da pesquisa realizada, cabe dizer que uma pandemia de grande magnitude implica em alterações psicossociais que podem ultrapassar a capacidade de enfrentamento da população afetada, e pode ser considerada, que toda a população sofre com medo, angústia e pânico em proporções diferentes. Dessa forma, é preciso, que haja uma construção de enfrentamento entre os diversos atores sociais incluídos nesse processo, ou seja, a população, as autoridades sanitárias e o poder público.

Nesse sentido, foi constatado através de pesquisas realizadas, que grande parte da população brasileira sofre com os efeitos secundários da pandemia, sendo eles provenientes da saúde mental, sendo a maior parcela desse público composto por 
mulheres, as quais já eram mais propensas antes mesmo da pandemia, a sofrer com o aparecimento de diversos fatores emocionais que atenuavam no comprometimento da saúde psicológica.

A partir do decreto de estado de calamidade no Brasil, gerado pela Covid-19, o Ministério da Educação criou mecanismos para ajudar a diminuir a disseminação do vírus e preservar a saúde dos profissionais da educação e dos alunos. Dentro desse processo de mudanças, esses profissionais compostos em sua grande maioria por mulheres, sentiram-se pressionadas a se adaptarem a nova forma de ensino e se sentiram inseguras, além de ter que realizar seu trabalho em uma dupla jornada.

E em decorrência desse processo de mudanças, pesquisas apontam que os profissionais que trabalham na educação estão com sua saúde mental prejudicada, e esse trabalho demonstra, que no momento em que a educação enfrenta um dos maiores desafios da história que é a pandemia de Covid-19, é possível que esses profissionais formados em sua grande maioria por mulheres, estejam tomados pela ansiedade e estresse, por sentirem suas rotinas modificadas.

Nesse sentido, é preciso pensar, planejar e, acima de tudo, implementar ações de suporte à saúde mental com atendimentos psicossociais mais frequentes a essas educadoras. E para que se faça isso é necessário que os cuidados sejam realizados de forma abrangente com a finalidade de atingir e beneficiar o maior número dessas mulheres, direcionados às especificidades das demandas.

Em suma, este trabalho conseguiu atender os objetivos esperados e com isso ser uma fonte de estudo para novas pesquisas envolvendo essa mesma linha de pesquisa. Assim, enfatizando, que a saúde mental principalmente voltada as mulheres que trabalham na educação, é de relevante importância, e precisa ser preservada, mesmo diante de tantos acontecimentos em tempos de pandemia.

\section{Referências}

Aquino, E. M. L. et al. (2020). Medidas de distanciamento social no controle da pandemia de covid 19 potenciais impactos e desafios no Brasil. Ciência \& Saúde Coletiva, Rio de Janeiro, v. 25, supl. 1, p.2423-2446.

Costa, A. T., Costa, S. T. H., Cardoso, N. J., Costa, A. J., \& Brito, D. M. (2020). A Saúde emocional dos professores durante a pandemia em tempos de aula remota 2020. VII Congresso nacional de educação: Educação como (re) Existência: mudanças, conscientização e conhecimentos. Ano 2020. ISSN: 23688829.

Ferreira, I. (2021). Mulheres foram mais afetadas emocionalmente pela pandemia. https://www.uol.com.br/vivabem/noticias/redacao/2021/02/14/mulheresforam-maisafetadas-emocionalmente-pela-pandemia.htm?cmpid.

Werneck, L. G., \& Carvalho, S. M. (2020). A pandemia de COVID-19 no Brasil: crônica de uma crise sanitária. Cad. Saúde Pública 36 (5) https://doi.org/10.1590/0102-311X00068820.

Kola, L. (2020). Global mental health and COVID-19. The Lancet Psychiatry, 7(8), 655-657. https://doi.org/10.1016/S2215-0366(20)30235-2

Lima, C. K. T. et al. (2020). The emotional impact of Coronavirus 2019-nCoV (new Coronavirus disease). In Psychiatry Research, $287(1)$ : 1-2.

Machado, D. B.; Teixeira, C. S. S.; Rocha, A. dos S.; Alves, F. J. O. COVID-19 e saúde mental: potenciais impactos e estratégias de atenção psicossocial. https://repositorio.ufba.br/ri/bitstream/ri/32942/10/vol2_cap10_COVID-19\%20e\%20a\%20sa\%C3\%BAde\%20mental.pdf

Barreto, M. L., Pinto Junior, E. P., Aragão, E., \& Barral-Netto, M. (org.). (2020). Construção de conhecimento no curso da pandemia de covid-19: aspectos biomédicos, clínico-assistenciais, epidemiológicos e sociais. Salvador: Edufba, 2020. v. 2. DOI: https://doi.org/10.9771/9786556300757.010.

Matta, G. C. et al. (2021). Os impactos sociais da Covid-19 no Brasil: populações vulnerabilizadas e respostas à pandemia. Série Informação para ação na Covid-19 | Fiocruz Language:Portuguese, DOI: https://doi.org/10.7476/9786557080320.

Miranda, T. S. et. al. (2020). Incidência dos casos de transtornos mentais durante a pandemia da COVID-19. Revista Eletrônica Acervo Científico / Electronic Journal Scientific Collection., v. 17. DOI: https://doi.org/10.25248/reac.e4873.2020

Organização Pan-Americana da Saúde (OPAS). (2021) Folha informativa COVID19 (doença causadapelonovocoronavírus). https://www.paho.org/bra/index.phpoption=com_content \&view=article \&id=6101: covid19\&Itemid=875

Organização Mundial da Saúde. (1997). Classificação Estatística Internacional de Doenças e Problemas Relacionados à Saúde - CID-10. (10Âa ed.) São Paulo: Editora da Universidade de São Paulo.

Pessa, V. E., Cruz Vaz CDL, Luz LS, Silva Pereira MF, Lazzerini M. COVID-19 among health workers in Brazil: the silent wave. J Glob Health. 2020; 10: 010379 
Research, Society and Development, v. 10, n. 11, e567101118268, 2021

(CC BY 4.0) | ISSN 2525-3409 | DOI: http://dx.doi.org/10.33448/rsd-v10i11.18268

Pinho, P.S., \& Araújo, T.M. (2012). Associação entre sobrecarga doméstica e transtornos mentais comuns em mulheres. Rev Bras Epidemiol, v. 15.

Qiu, J. et al. (2020). A nationwide survey of psychological distress among Chinese people in the COVID-19 epidemic: Implications and policy recommendations. General Psychiatry, 33(2), https://doi.org/10.1136/gpsych-2020-100213

Nova Escola. (2021). A situação dos professores no período da pandemia. https://www.andes.org.br/diretorios/files/renata/junho/ne-pesquisa-professor-final1.pdf.

Silva, A. F., Estrela, M. F., Lima, S. L., \& Abreu, A. T. C. (2020). Saúde mental de docentes universitários em tempos de pandemia. Physis, 30 (02). DOI: https://doi.org/10.1590/S0103-73312020300216.

Souza, A. S. R., Souza, A. F. G., \& Praciano, F. A. G. (2020). A saúde mental das mulheres em tempos da COVID-19. Rev. Bras. Saúde Mater. Infant. 20(3). DOI: https://doi.org/10.1590/1806-93042020000300001

Thapa, S.B., Mainali, A., Schwank, S.E., \& Acharya, G. (2020). Maternal mental health in the time of the COVID-19 pandemic. Acta ObstetGynecol Scand.

Xiang, Y.T. et al. (2020). Timely mental health care for the 2019 novel coronavirus outbreak is urgently needed. Lancet Psychiatry. [Internet]. 7(3). https://www.thelancet.com/action/s howPdf?pii=S2215- 0366\%2820\%2930046-8 